\title{
4
}

\section{MOOSE: A method designed for ease of maintenance}

\author{
R. I. Ferguson, N. F. Parrington and P. Dunne
}

University of Sunderland, School of Computing and Information Systems, Priestman Building, Green Terrace, Sunderland, Tyne/Wear, SR1 3SD, UK ,Tel. +44 091 5152508, Fax.+44091 5152781, Email - i.ferguson@sunderland.ac.uk

\begin{abstract}
The problem of change as manifested in perfective and adaptive maintenance is presented along with some of the qualities that software must possess in order to minimise the effects of change. These qualities can be provided by the Object Oriented paradigm. This paper describes the current work on a Method for Object Oriented Software Engineering (MOOSE) that has ease of maintenance and re-use as primary design objectives. A prototype CASE tool to support the method has also been developed: details and proposals for its re-engineered capabilities are given. No attempt is made to completely define the method, instead a general description of its features, particularly those pertinent to maintainability, is presented.
\end{abstract}

keyword codes : D.1.5; D.2.2; D.2.7

keywords : object-oriented programming; tools and techniques; distribution and maintenance

\section{THE PROBLEM}

Lehman's first law states that any system's requirements will change over time and that for the software to remain useful, it too must change. [1]. The Software Engineer is thus faced with the prospect, that no matter how well the stages of elicitation, specification, analysis and design are performed, a significant amount of the system produced will need re-engineering over its lifetime.

Software Maintenance activities are frequently categorized (following Swanson [2]) as "Perfective" - consequent on change of requirement, "adaptive",- due to change in operating environment - "corrective" - bug fixing or "preventative" [3].

The key factors influencing maintainability are complexity and modularity [4]. Complexity is a measure of how difficult a piece of code it to understand. If a piece of software has been refined into a set of smaller sub-problems then it is said to be modularised. If the individual modules have low external coupling and high internal cohesiveness, then the system will be easier to maintain as the effect of changing any one piece of code will be limited to the module in which the change occurs. Another factor affecting the maintainability of a system is the ease with which a programmer can maintain a mental model of what the system does, and how it is built.

\section{A SOLUTION}

One paradigm that claims to improve these factors is the Object Orientation (OO).Rooted firmly in modularity, its modelling approach is closer to human ways of reasoning than procedural methods [5], and it isolates the effects of change through its use of information hiding[6]. Object orientation gives a system the flexibility required by Lehman's 1st law. Requirements do not have to be artificially frozen at design time (as assumed by the traditional software development lifecycle).They may evolve with time and the system development process needs to take this into account. 


\section{METHOD - MOOSE}

A method is currently being developed at the University of Sunderland to take advantage of the object oriented paradigm. It remains flexible enough that engineers can work in their own manner, yet provides sufficient constraints to facilitate the production of high quality code. It uses prototyping to allow ease and accuracy of requirement elicitation, while the use of formal specification ensures that there exists an unambiguous definition of each module's purpose. It rigorously employs the ideas of modularity but still allows an experimental programming approach to be taken where required. The method is known as MOOSE - Method for Object Oriented Software Engineering

\section{1 - Fundamental concepts}

MOOSE is closely linked with its supporting CASE tool, JACI (Just Another CASE Implementation). The notations used by MOOSE have been designed with tool assistance in mind and would prove awkward to handle without the aid of the tool. Moreover, inconsistencies in the underlying database can be introduced by working on a system with a tool that is not part of JACI. This must not be permitted, as it is from this database that the executable code is generated.

\section{2 - Underlying Concepts}

MOOSE is modular. Every piece of software built via the method is treated as a module, whether it is a complete air traffic control system or a three line routine. The basic unit of modularity is the OO "class" Each module can be treated separately from any other module in the system. When necessary, a module can consist of, or use, smaller sub-modules which can likewise be refined, ultimately into problems of a manageable size. This process of refining can therefore be described as recursive and it produces a hierarchy of modules. In fact, several different hierarchies are produced depending on the relationships between modules.

One concept crucial to MOOSE and the supporting tool is that of the module framework. This isn't a code template; rather it is a database schema with fields for storing all the information that is required to completely describe one module.:

\section{Table 1: MOOSE module framework 'fields'}

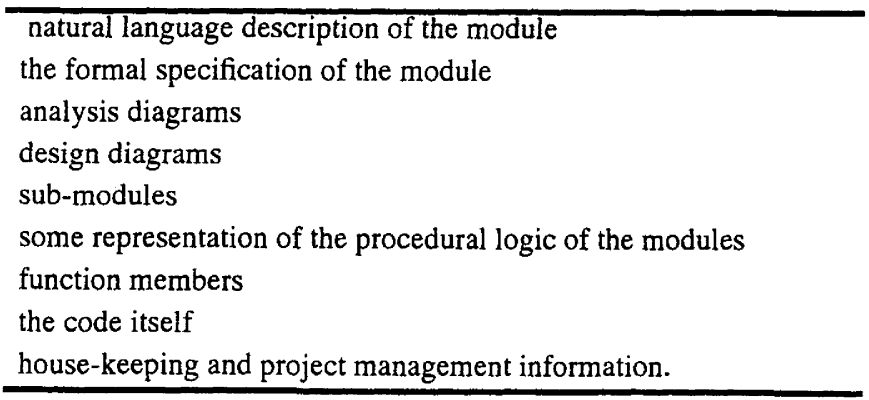

All stages (activities) of MOOSE result in some deliverables. It is these deliverables that are stored in the fields defined by the module framework. Thus if a framework for a system level object is complete., then a complete specification for a whole system will exist. In fact, this toplevel framework has a physical counterpart in the tool's database.

\section{3 - Components}

The MOOSE method is built on "approaches", "activities" and "techniques". 


\subsection{1 - Approaches}

The approaches available to a software engineer are "top-down", "prototyping" and "experimental-programming" which represent the three main ways in which software can be constructed [7].

One of the reasons that three different approaches can be taken, is that deliverables arise from "the wrong activity". For instance, if an experimental-programming approach is being taken to build a module, when the module is complete, a design must exist for it, which can be "backfilled" into the module framework design diagram field. In many cases it should be possible to automate large parts of this process..

\subsubsection{1 - Top-Down}

The top-down approach is the classical method of software production. It involves starting with a rough description of a problem and gradually refining that description into smaller subproblems which are then repeatedly refined until each problem is codeable. Associated with this approach is the idea of sequentially performing analysis, design and coding with each stage not commencing until the previous one has been completed. MOOSE permits this approach but allows backtracking and iteration of activities.

\subsubsection{2 - Prototyping}

MOOSE encourages the use of three types of prototyping[8];throwaway, evolutionary and incremental. Prototyping is used primarily to identify user interaction with the system and to map interface items (menus, buttons, etc.) onto classes that can implement that means of interaction. Tool support for this is available via the Tcl/Tk prototyping langauages.[9]

\subsubsection{3 - Experimental programming}

Experimental programming has been referred to as programming without a specification. [10]. It is particularly useful for designing new algorithms, but likely to cause maintenance problems if the code developed is not subsequently documented. MOOSE allows this approach with the caveat that it is best done in conjunction with the JACI CASE tool, which gives significant help with retro-documenting.

\subsubsection{4 - Mixing approaches}

Within software development, different approaches are appropriate depending upon the level of the desired system that is being worked on. A "top- down" approach is more appropriate for high-level (top of system) work - for things that might be termed "application objects". Experimental-programming is better for code closer to machine level - "utility objects."

Perhaps the most powerful approach is to combine approaches. This combines the structure of top-down with the exploratory power of experimental programming. This would permit (for instance) the use of a top down approach to develop a basic understanding of the system, the use of prototyping to ensure correct user interaction and the use of experimental programming to get the latest sort-routine working at optimum speed.

\subsection{2 - Activities}

The three activities of the method are "analysis", "design" and "coding". These are not the usual distinct phases of traditional software development but form a continuum, although each activity still has associated deliverables. This continuum arises for 2 reasons, i) the OO paradigm uses the same modelling concepts throughout development, ii) MOOSE uses a unifying notation which is applicable throughout the lifecycle.

\subsubsection{1 - Analysis}

Analysis in MOOSE is achieved by identifying the entities that make up a system and the rela- 
tionships between them. The relationships are then categorised and refined. The data necessary to represent those entities are then added followed by the operations that can be performed on them. Each identified entity is then refined recursively. Analysis uses a natural language description of the problem (PEP) as its starting point. The entities identified from this description are then shown on a form of modified Entity-Relationship diagram [11] known as the analysis diagram.

The relationships between the objects that have been identified are then examined. Relationships fall into four significant categories based on those used by Booch [12]: "has_a", "is_a_kind_of", "contains" and "uses". A hierarchy of classes is associated with each category. The cardinality of each relationship (1:1, 1:M, M:M) is identified.

\subsubsection{2 - Design}

In the design activity, each module identified on the analysis diagram is converted to an individual design diagram. Modelling of things "systemic" [13] to this level then commences. This includes: addition of data members to classes, addition of function members, derivation of a formal specification,algorithm design, addition of parameter passing information

\subsubsection{3 - Coding}

If the "top-down" approach of MOOSE has been used exclusively, a complete set of design diagrams will exist following completion of the design activity. The diagramming notation has been so designed as to reduce coding to a mechanical process. Although it is not yet implemented we expect that to a large extent, code generation can be automated. Conversely, if an exclusively experimental-programming approach has been taken, coding may well be the first activity undertaken.By using the editors built into $\mathrm{JACl}$, the code is entered directly into the system database. From there, it can be reverse engineered into diagrammatic form, in real time thus providing design documentation 'on-the-fly'.

\subsection{3 - Notation - analysis diagrams}

Analysis diagrams are closely related to Entity-Relationship diagrams. Their main features are as follows:-

\subsubsection{1 - Modules}

The presence of a module simply indicates the existence of an entity in the real-world system being modelled. These are designed to look like the "class" of the design notation. Each module must have a unique name; using JACI, they can be expanded to their corresponding "class" diagram at the click of a button.

\subsubsection{2 - Relationships}

Relationships indicate a link of some sort between two entities. Each relationship must have a name. Conventional "crows feet" indicate the cardinality of the relationship.

\section{Fig. 1-Modules and relationships}
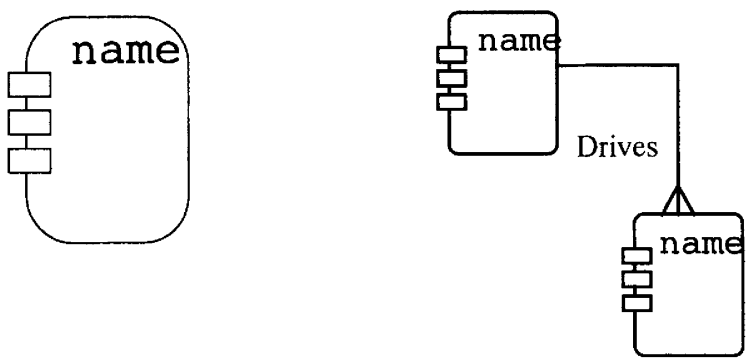


\subsubsection{3 - Categories}

Relationships fall into 4 categories, each of which is implemented in a different way. An "is_a kind_of" relationship indicates inheritance of some kind is required. A "contains" relationship is implemented using a container class. "Has_a" relationships indicate participation in a whole/part hierarchy. The "uses" type of relationship implies the use of pointers to maintain links between instances of two classes. From the identification of these relationships part of the design can be inferred automatically The identification of "is_a_kind_of" and "has_a" relationships leads to the construction of hierarchies of entities. It can be useful to identify the existence of such structures on separate analysis diagrams.

\subsection{4 - Notation - design diagrams}

A single design can be made up of a series of design diagrams. For every entity identified on an analysis diagram, a corresponding diagram should exist.Design diagrams contain a lot of "link" information (which we have dubbed "hypercase links"), between diagrams, subsections of diagrams and other parts of the MOOSE database. Modules (classes) are shown thus:-

\section{Fig. 2 - Classes / datamembers / function members}
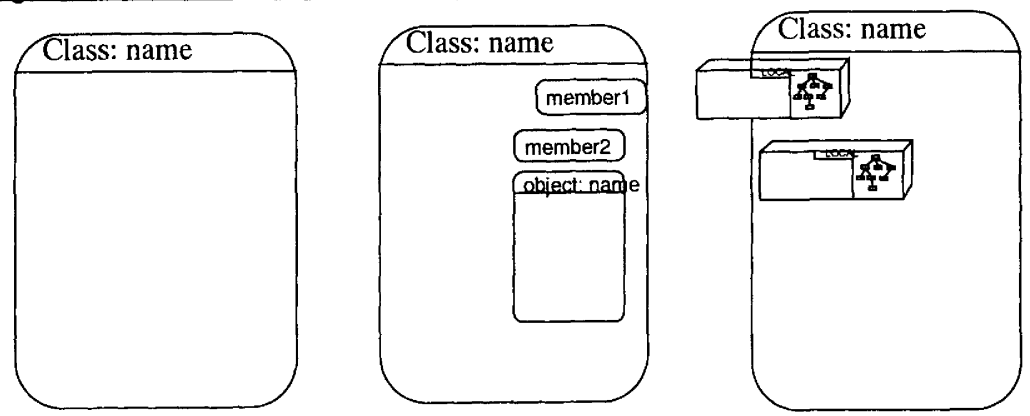

\subsubsection{1 - Data members}

Data members can be added to a class by placing them on the right hand side of the owning classes' bounding box. The member may be of either object or non-object type. The visibility of a member is indicated by its position: inside the class - private, touching the edge of the class public. The C++ "protected" visibility is dealt with below (Section 3.3.4.7 - "Inheritance")

\subsubsection{2 - Function members}

Function members are added on the left hand side. Implementation functions appear entirely within the bounding rectangle of the owning class. "Policy" methods are shown partially protruding from the bounding box.

\subsubsection{3 - Function icon}

The function member "icon" has several parts: i) name of the function (or method), ii) a local variable area/ "landing area" for formal parameters.(See "function calls" below), iii)a code icon. In JACI, access to the code representations are accessed via "clicking" on this icon. The impossibility of having the code represented directly in this space is a consequence of designing the notation with tool support in mind.

\subsubsection{4 - Parameters}

Three types of formal parameter are supported, based on the Ada standard parameter declaration; .in, out, in/out 


\section{Fig. 3 - Functions / Parameters}
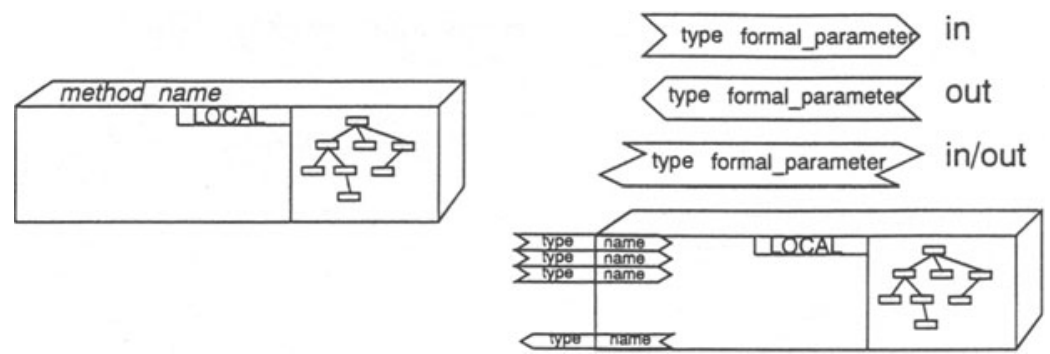

\subsubsection{5 - Procedural logic}

Procedural logic can be represented in three ways in MOOSE; pseudocode; JSP and directly in the target language code. (Although MOOSE itself is language independent.)The code icon is shown here as 'exploding' to a JSP diagram editor. A prototype diagram-text translator has been implemented allowing the simultaneous display/editing of code and JSP.

\section{Fig. 4 - Procedural logic / Function Calls}
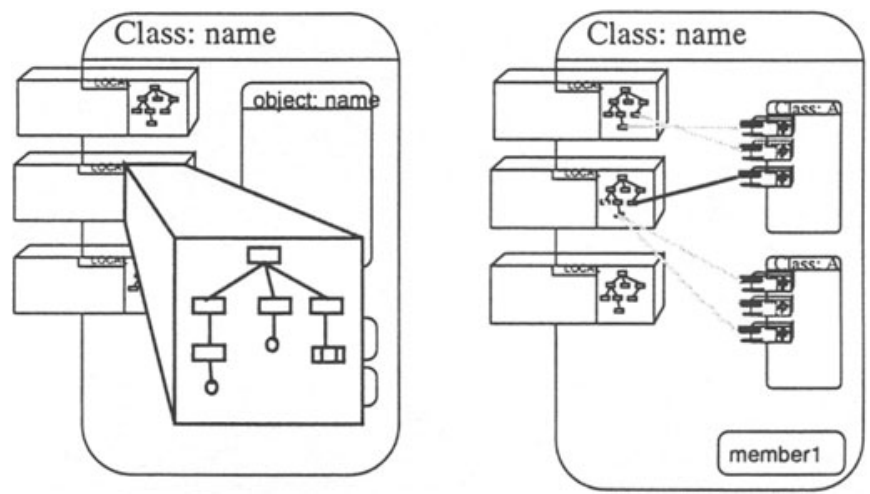

\subsubsection{6 - Function calls (message passing)}

Function calls ("message passing" is the $\mathrm{OO}$ term) take place between methods. They originate with the methods of the class being designed and are targeted on methods of member data. In MOOSE, they are simply shown as un-annotated lines. If every possible function call were shown simultaneously on a design diagram the result would be an extremely cluttered diagram. To avoid this, the number of links shown at any one time are limited by visibility controls.

\subsubsection{7 - Inheritance}

On a class' defining design diagram, any members which are defined as "protected" (accessible only to the methods of descendant inheriting classes) are shown in a different colour to "private" or "protected" members. In any descendant class, only the public members inherited will be displayed. Inheritance is shown in the following fashion. The idea being that descendants appear to be "built upon" their ancestors. 
Fig. 5 - Inheritance

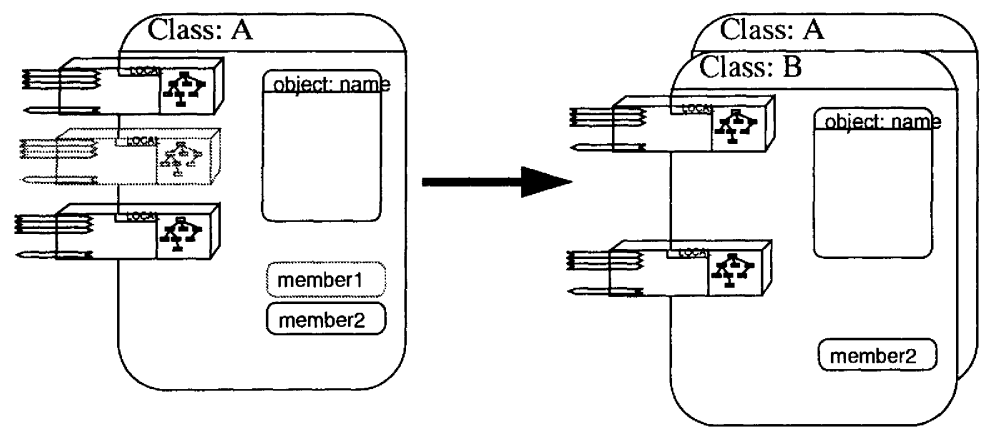

\subsubsection{8 - Genericity}

No special graphic notation is required to define a generic (template) class. Any variables (usually parameters of the classes method) that are of unknown type at design time are represented on the diagram prefixed with a designatory identifier surrounded by "<" and ">" characters in much the same manner as they are treated in $\mathrm{C}++$. e.g. $\langle\mathrm{T}>$ my_variable.

\subsubsection{9 - Container classes}

Some representation of the containment of data in structures like arrays, queues etc. was desirable to match the programmers metal model of the implementation. The following notation allow messages to be sent to the container class and the data contained within it. The container class can be defined using the elements of notation already described; however when a container appears in a diagram as a member object, the following "square box" convention is required. This way both the methods of the container class and the contained class are "exposed" for potential use the owning classes methods.

\section{Fig. 6 - Containers}

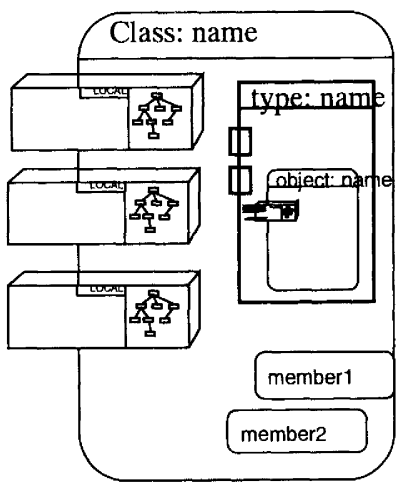

\subsubsection{0 - Tool Support}

MOOSE will be supported by an integrated CASE toolset based on a 2 tier PCTE database. Named JACI, the toolset currently exists in prototype form only. When complete, it will include editors to cope with all the notations of MOOSE. A network of "hypercase" links allow navigation between the different representations of entities. The links reach to every possible representation of any given entity in the database and between related entities. The common database should also allow the integration of facilities such asthe AI based NLP tools, the expert "method navigator", 
automatic translation between representations where possible. and the browser/librarian allows perusal of existing modules facilitating reuse.As a consequence, an engineer should rapidly be able to access all of the sources of information from which a given module was produced.

\section{CONCLUSION}

There are four main reasons why MOOSE produces code that is highly maintainable: i) the generated code is well modularised ii) the modelling method is intuitive and matches human thought process, iii) genericity/inheritance and the library encourage re-use iv) documentation availability. Provided the whole database is committed to the re-use library each module will always have its specification and design documentation available.

\section{FUTURE WORK}

Since the analysis and design diagrams form such a complete specification of a module's properties and behaviour, it has been proposed, but not investigated, that the diagrams could actually be executed, forming the basis of a truly "visual" language. Such a language, based on a readily understandable notation, could have major consequences for maintainability.

\section{References}

1 Lehman, M.M. and Belady L. "Program Evolution. Processes of Software Change." London, Academic Press

2 Swanson, E.B. "The Dimensions of Maintenance", Proc. 2nd Intl. Conf. Software engineering, IEEE, Oct 1976, pp. 492-497

3 Pressman, R.S. (1992) Software Engineering: A Practitioners Approach 3rd Ed, p. 664, McGraw-Hill, NY, USA

4 Curtis, B. and Sheppard, S.B. (1979) Identification and validation of quantitative measures of the psychological complexity of software. Software Management Research Report, General Electric

5 Wiener, R.S. and Pinson, J.P., (1988), "An Introduction to Object-Oriented Programming and C++", Addison-Wesley, p. iii

6 Meyer, B., (1988) “Object-oriented Software Construction”, Prentice-Hall, pp7-25, “Aspects of Software Quality"

7 Pressman, R.S. (1992) Software Engineering: A Practitioners Approach 3rd Ed, p. 22-37, McGraw-Hill, NY, USA

8 Ince, D.,(1993) "Prototyping" in "Software Engineers Reference Book" ed. McDermid, J., Butterworth-Heineman

9 Ousterhout, J., "Tcl and the Tk Toolkit",(1994), Addison-Wesley

10 McDermid J. and Rook, P.,(1991) "Software Development Process Models" in "The Software Engineers Reference Book", ed. McDermid, J., Butterworth-Heineman

11 Chen. P., "The Entity-relationship Model -towards a unifying view of data", ACM Transactions on Data Base Systems, Vol.1, 1, March 1976, pp 9-36

12 Booch. G. "Object Oriented Analysis and Design" 2nd Ed, Benjamin-Cummings, ch.5 pp176-180

13 Checkland. P.,(1981), "Systems Thinking, Systems Practice", Wiley 